Draft OF MAY 18, 2018

Preprint typeset using $\mathrm{IAT}_{\mathrm{E} X} \mathrm{X}$ style emulateapj v. 12/16/11

\title{
CATSHTM - A TOOL FOR FAST ACCESSING AND CROSS-MATCHING LARGE ASTRONOMICAL CATALOGS
}

\author{
Maayane T. Soumagnac ${ }^{1} \&$ Eran O. OfeK $^{1}$ \\ Draft of May 18, 2018
}

\begin{abstract}
Fast access to large catalogs is required for some astronomical applications. Here we introduce the catsHTM tool, consisting of several large catalogs reformatted into HDF5-based file format, which can be downloaded and used locally. To allow fast access, the catalogs are partitioned into hierarchical triangular meshes and stored in HDF5 files. Several tools are provided to perform efficient cone searches at resolutions spanning from a few arc-seconds to degrees, within a few milliseconds time. The first released version includes the following catalogs (by alphabetical order): 2MASS, 2MASS extended sources, AKARI, APASS, Cosmos, DECaLS/DR5, FIRST, GAIA/DR1, GAIA/DR2, GALEX/DR6Plus7, HSC/v2, IPHAS/DR2, NED redshifts, NVSS, PanSTARRS1/DR1, PTF photometric catalog, ROSAT faint source, SDSS sources, SDSS/DR14 spectroscopy, Spitzer/SAGE, Spitzer/IRAC galactic center, UCAC4, UKIDSS/DR10, VST/ATLAS/DR3, VST/KiDS/DR3, WISE and XMM. We provide Python code that allows to perform cone searches, as well as MATLAB code for performing cone searches, catalog cross-matching, general searches, as well as load and create these catalogs.
\end{abstract}

\section{INTRODUCTION}

In the past three decades, the emergence of catalog services like SIMBAD ${ }^{2}$ (Wenger et al. 2000), VizieR ${ }^{3}$ (Ochsenbein et al. 2000), the NASA Extragalactic Database (NED) ${ }^{4}$ and MAST ${ }^{5}$ have had, and continue to have, an enormous impact on astronomical research. These services are being used extensively by the astrophysics community and are used in a large fraction of articles. Nevertheless, one obvious limitation of all these services is that the Internet connection limits the speed of search. This is an obstacle for some applications requiring very fast access to large catalogs. Examples of such applications are cross-matching multiple large catalogs (e.g., Malkov et al. 2012, Ogle et al. 2015), and vetting transient candidates detected by synoptic surveys. For example, surveys like Pan-STARRS (Chambers et al. 2016) and the Palomar Transient Factory (Law et al. 2009 generated a large number of transient candidates which have to be cross-matched with multiple catalogs in order to be vetted, classified and followed up.

Cross-matching tools developed in the last years include the web-based tools CDS-Xmatch (Pineau et al. 2011) and ARCHES (Motch \& Arches Consortium 2015), or local command line tools like TOPCAT ('Taylor 2005), STILTS (Taylor 2006) and $C^{3}$ (Riccio et al. 2017) which allow to overcome some of the speed limitations of the web-based applications. Another simple solution is to use local relational databases. Indeed, with proper indexing (e.g., Hierarchical Triangular Mesh (HTM); Szalay et al. 2007), Structured Query Language (SQL) queries

\footnotetext{
${ }^{1}$ Benoziyo Center for Astrophysics, Weizmann Institute of Science, 76100 Rehovot, Israel,

Corresponding author:

Maayane T. Soumagnac, maayane.soumagnac@weizmann.ac.il

2 http://simbad.u-strasbg.fr/simbad

3 http://vizier.u-strasbg.fr

4 https://ned.ipac.caltech.edu

5 https://archive.stsci.edu/astro
}

on such database are relatively fast. In this paper we present a simple and - at least from our experience faster alternative.

We provide a set of large catalogs stored in HDF5 files ${ }^{6}$ HDF5 is a data model, library and file format for storing and managing data. It supports an unlimited variety of data types and is designed for flexible and efficient $\mathrm{I} / \mathrm{O}$ and for high volume and complex data. Furthermore, HDF5 tools are available in many computer languages. The data storage methodology we use is designed to provide good performances both for small size (i.e., a few arcsec) and large size (i.e., deg) cone searches. In addition to the formatted catalogs, we provide a set of tools to perform fast cone search, serial search, catalog crossmatching and catalog generation.

The structure of this paper is as follows. In 82 , we detail the structure and format of the catalogs. In 93 we list all the catalogs currently available and present the codes we provide for fast access to these catalogs. We compare our tool to other existing tools and discuss our results in 4 .

\section{THE DATA FORMAT}

The efficiency of catsHTM lies in three aspects: (1) the way the data is partitioned into files; (2) the way the data is stored in these files; and (3) the data indexing.

The data of each catalog is partitioned in the following way. We divide the celestial sphere using a Hierarchical Triangular Mesh (HTM) quad-tree (Szalay et al. 2007 Kunszt et al. 2000). This method is based on a recursive subdivision of the celestial sphere into spherical triangles 7 of similar shapes, called trixels. The HTM method of dividing the sphere is particularly good at supporting searches at a wide range of resolutions (hemispheres to arc seconds). In our case, depending on the size of the

\footnotetext{
6 https://support.hdfgroup.org/HDF5/

7 A spherical triangle is a polygone of which the edges are segments of three great circles.
} 


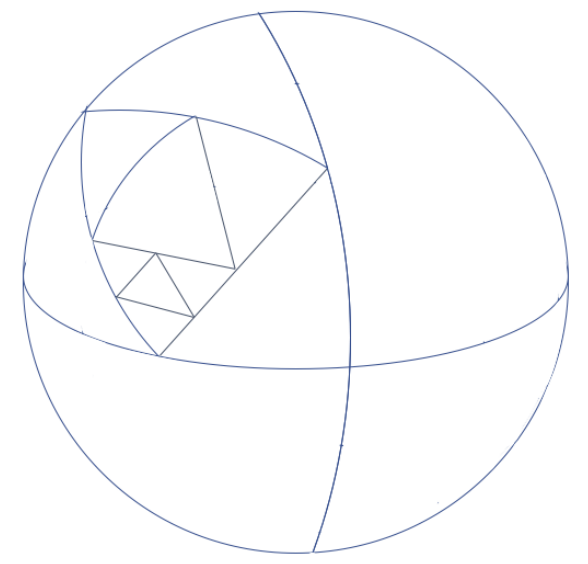

FIG. 1.- Illustration of the recursive decomposition of the sphere into trixels, up to level 3. The trixels are spherical triangles: polygons of which the edges are segments of three great circles. Each trixel has four "sons" (i.e.; quad tree).

catalog, the level of the HTM (i.e., the number of subdivisions necessary to create the smallest trixel) varies between six and nine. The amount of levels we chose provides good efficiency for cone searches at resolutions spanning from a few arc-seconds to a degree - i.e., the typical resolutions usually used for astronomical applications. Each trixel in the quad-tree is allocated a number, which is referred to as the trixel index throughout this paper. In figure 1, we show an illustration of the recursive decomposition of a sphere into HTM trixels.

To store the data of the partitioned catalogs, we use datasets, one of the object types available within the HDF5 data mode ${ }^{6}$ A dataset is simply a multidimensional array and a HDF5 file can contain multiple datasets.

For each trixel, we create a pair of datasets:

1. The trixel-dataset, containing the actual catalog data that are within the trixel. Only highest level trixels are populated (and exist). The data in each trixel is sorted by declination.

2. The index-dataset, containing a two column matrix. The first column is the line number in the trixel-dataset and the second column is the corresponding declination. This data exist in steps ranging from 30 to 300 lines and can be used in some cases to expedite a search within a trixel. This is mainly used for catalogs cross-matching.

These pairs of datasets are then stored, in groups of 100 (by default), in HDF5 files.

For each partitioned catalog, we create an additional HDF5 file, called the HDF5 index file, which contains the information needed in order to access the relevant trixels, when making a cone search. The HDF5 index file contains as many lines as trixels in the tree. Each line corresponds to one trixel and contains: the index of the trixel; the indexes of the parents and sons trixels; the sky coordinates (latitude and longitude) of the poles of the great circles that defines the trixel; the number of sources it contains ( $\mathrm{NaN}$ if the trixel does not belong to the highest level in the tree). The HDF5 index file allows to perform an efficient tree search for all the trixels
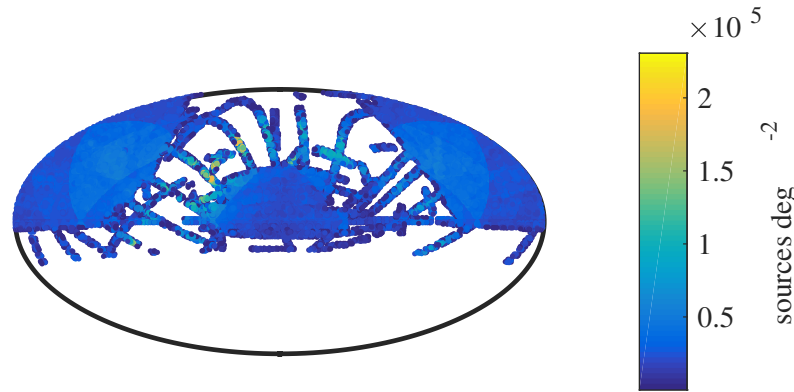

FIG. 2.- Source density in the SDSS-DR10 catalog as plotted using the catsHTM.plot_density function.

that intersect - or are contained within - a given cone. Finally, we also keep a file containing additional meta data, such as the catalog column names and units.

To summarize: each catalog is partitioned into trixels. For each partitioned catalog, the following files exist: (1) HDF5 files containing datasets where the catalog data can be stored (2) a HDF5 file called the index file, where the index and sky coordinates of each trixel are stored (3) a file containing meta data on the catalog.

In Table 1, we present the default files and datasets naming formats we used. Additional documentation and examples are available on-lin $\AA^{8}$.

\section{AVAILABLE CATALOGS AND CODES}

The catalogs currently available are listed in Table 2 and the list will be updated regularly in the on-line documentation 8 . The catsHTM directory containing the HTM/HDF 5 catalogs requires $\sim 2.1 \mathrm{~TB}$ of disk space. Download instructions as well as links to the code used to create those catalogs can be found in the on-line documentation 8

We provide two sets of codes to access the formatted catalogs listed in Table 2 .

1. A Python code 9 that provide cone search functions;

2. A MATLAB code $\AA^{10}$ that can perform a variety of tasks in addition to cone search, described in the on-line documentation 8 general searches, cross-matching, plotting, loading catalogs and generating new catalogs. For example, in Figure 2 we show a sky map, generated by one of these functions, that presents the source density in the SDSS catalog.

Currently, the Python code is compatible with both Python 2 (higher than 2.7.10) and Python 3 and requires a small amount of standard basic packages (numpy, scipy, math and h5py). The MATLAB code is available as part of the MATLAB Astronomy \& Astrophysics Toolbox ${ }^{11}($ Ofek 2014), and has been tested with MATLAB R2016b. These requirements will be updated, if needed, in the online documentation, as future versions of catsHTM are released.

8 https://webhome.weizmann.ac.il/home/eofek/matlab/doc/ catsHTM.html

9 https://github.com/maayane/catsHTM

10 https://webhome.weizmann.ac.il/home/eofek/matlab/doc/ install.html

${ }^{11}$ https://webhome.weizmann.ac.il/home/eofek/matlab 
TABLE 1

Default NAMing Format

\begin{tabular}{lll}
\hline \hline \multicolumn{1}{c}{ Object } & \multicolumn{1}{c}{ Default naming format } & \multicolumn{1}{c}{ type } \\
\hline Trixel-dataset & htm_\%06d & HDF5 dataset \\
Index-dataset & htm_\%06d_Ind & HDF5 dataset \\
HDF5 files containing the trixel-datasets and the index-datasets & <CatBaseName>_htm_\%06d.hdf5 & HDF5 file \\
HDF5 index file & <CatBaseName>_htm_Ind.hdf5 & HDF5 file \\
Catalog metadata (e.g., column names) & <CatBaseName>_htmColCell.mat & .mat file \\
\hline
\end{tabular}

Note. - " $<$ CatBaseName $>$ " is the catalog name (see Table 2). The default number of pairs of trixel- and indexdatasets in each HDF5 file is 100. In the HDF5 index file, the catalog file name index is rounded such that the last two significant digits are always 0 (in case of 100 trixels per HDF5 file).

TABLE 2

List of AVAILABLE CATALOGS (UPDATED IN THE ON-LiNE DOCUMENTATION)

\begin{tabular}{|c|c|c|c|c|}
\hline CatBaseName & Catalog & $N_{\text {src }}$ & $N_{\text {col }}$ & Reference \\
\hline TMASS & 2MASS & 470992608 & 10 & Skrutskie et al. $(2006)$ \\
\hline TMASSxsc & 2MASS extended sources & 1647599 & 15 & Jarrett et al. $(2000)$ \\
\hline AKARI & AKARI & 870973 & 9 & Ishihara et al. (2010) \\
\hline APASS & APASS & 55395532 & 19 & Henden et al. (2009) \\
\hline Cosmos & Cosmos & 438226 & 27 & Capak et al. (2007) \\
\hline DECaLS & DECaLS/DR5 & 679250688 & 26 & http://legacysurvey.org/decamls/ \\
\hline FIRST & FIRST & 946432 & 14 & Becker et al. (1995) \\
\hline GAIADR1 & GAIA/DR1 & 114268060 & 8 & Gala Collaboration et al. (2016) \\
\hline GAIADR2 & GAIA/DR2 & 1692967552 & 27 & Gala Collaboration et al. (2018) \\
\hline GALEX & GALEX/DR6Plus7 & 165794048 & 9 & Mlartin et al. (2005) \\
\hline HSCv2 & Hubble Source Catalog HSC/v2 & 318758784 & 15 & Whitmore et al. (2016) \\
\hline IPHAS & IPHAS/DR2 & 205773248 & 17 & Barentsen et al. \\
\hline IRACgc & Spitzer/IRACgc & 1065565 & 15 & Ramirez et al. (2008) \\
\hline $\mathrm{NEDz}$ & NED redshifts (2018 May 2 version) & 7154168 & 8 & Helou et al. (1990); Mazzarella \& NED Team (2017) \\
\hline NVSS & NVSS & 1773486 & 12 & Condon et al. $(1998)$ \\
\hline $\mathrm{PS} 1 \mathrm{~b}$ & Pan-STARRS1/DR1 & 2612435712 & 41 & Chambers et al. (2016) \\
\hline PTFpc & PTF photometric catalog & 21167678 & 14 & Ofek et al. (2012) \\
\hline ROSATfsc & ROSAT faint source & 105924 & 21 & Voges et al. (2000) \\
\hline SAGE & Spitzer/SAGE & 9094829 & 16 & Meixner et al. (2006) \\
\hline SDSSDR10 & SDSS sources & 447279840 & 16 & Âhn et al. (2014) \\
\hline SpecSDSS & SDSS/DR14 spectroscopy & 4311570 & 32 & Aboltathı et al. 2017 ) \\
\hline UCAC4 & UCAC4 & 113780216 & 45 & Zacharias et al. 2013$)$ \\
\hline UKIDSS & UKIDSS/DR10 & 79333520 & 38 & Lawrence et al. $(\overline{2007})$ \\
\hline VSTatlas & VST/ATLAS/DR3 & 106556464 & 14 & Shanks et al. (2015) \\
\hline VSTkids & VST/KiDS/DR3 & 48735816 & 21 & de Jong et aI. (2017) \\
\hline WISE & WISE & 563908224 & 37 & Wright et al. $(2010)$ \\
\hline XMM & XMM & 727790 & 14 & Rosen et al. $(2016)$ \\
\hline
\end{tabular}

NoтE. - List of the catalogs available at the date of submission (this list will be regularly updated in the on-line documentation). In some cases, there are small discrepancies in the number of sources (typically $<10^{-5}$ ) between the version available on VizieR and our HDF5 version. Some of these differences are due to problems in the ingestion process and this will be fixed in future releases.

${ }^{a}$ A newer version of the APASS catalog will be included in one of the next releases.

${ }^{\mathrm{b}}$ Sources detected in the stacked images above a certain signal-to-noise ratio; the current version of the HDF5 catalog has some missing regions below $\delta=0$.

\section{RESULTS AND DISCUSSION}

Compared to one specific relational database SQL searches, we find our code to be about one order of magnitude faster for cone searches. A 10 arcsec radius cone search, on a random celestial position, using a Xeon(R) CPU E5-2670 v3 at 2.30 GHz machine takes on average about $1-4 \mathrm{~ms}$ using the MATLAB tool and $1-40 \mathrm{~ms}$ using the Python tool. A 1000 arcsec radius cone search on the same machine takes on average $4-300 \mathrm{~ms}$ using the MATLAB tool and 2-600 ms using the Python tool, where the exact time depends on the catalog.

Our code can also be used to efficiently cross-match catalogs and perform general searches. These options can use multiple processors. The cross-matching is performed by loading a single trixel dataset from the first catalog into memory and cross-matching it only with sources that are found in overlapping trixels from the second catalog. For example, using 24 processors, crossmatching of the APASS catalog against itself takes about $160 \mathrm{~s}$ while cross-matching the 2MASS catalog against the WISE catalog takes about 53 min (without dumping the results). The cross-matching tool is work in progress. In particular, it is currently only available in MATLAB and will be available in Python in future releases of catsHTM. In the future, we plan to extend the format for multipleepoch catalogs, to add new catalogs and to provide catalogs of cross-matchings between all the catalogs.

Making a fair comparison between the performances of catsHTM and those of other available tools is a complex task, because catsHTM comes with formatted catalogs.

Web-based tools like OpenSkyQuery (NietoSantisteban et al. 2006) and CDS-Xmatch (Pineau 
et al. 2011), as well as tools with a strong graphical component such as TOPCAT (Taylor 2005) consist of portals which have the disadvantage of being relatively hermetic to the user. The need to allow access to multiple users comes with its own set of compromises: CDS-Xmatch limits both the disk space and computation time available to the users.

On the contrary, catsHTM has been designed as a stand-alone tool, to avoid the above disadvantages and allow high flexibility. The simple cone search python and MATLAB codes can be easily edited according to the user's specific needs and easily integrated in the user's own pipelines. The all-in-one structure of the code and catalogs allows the user to run jobs on his own computer and without relying on internet connection. The $C^{3}$ tool (Riccio et al. 2017) has been designed in this spirit, but focuses on cross-matching, whereas catsHTM primary task is to perform efficient cone-search queries.

Perhaps the existing tool closest to what catsHTM is trying to achieve is STILTS (Taylor 2006) which, on the one hand, is a powerful stand-alone tool, free of the disadvantages of web-page applications, and on the other hand offers a large variety of catalog-handling functionalities, including cone search.

catsHTM is unique in the sense that it is not limited to the search and cross-matching tools it offers. These tools come together with a large - and constantly updated set of astronomical catalogs, formatted into the binary HDF5 format.

E.O.O. is grateful for the support by grants from the Israel Science Foundation, Minerva, Israeli ministry of Science, the US-Israel Binational Science Foundation, and the I-CORE Program of the Planning and Budgeting Committee and The Israel Science Foundation.

M.T.S. acknowledges support by a grant from IMOS/ISA, the Ilan Ramon fellowship from the Israel Ministry of Science and Technology and the Benoziyo center for Astrophysics at the Weizmann Institute of Science. M. T. S would also like to thank Steve Schulze, Barak Zakay, Ronen Tamari and Adam Rubin for useful discussions.

\section{REFERENCES}

Abolfathi, B., Aguado, D. S., Aguilar, G., et al. 2017, ArXiv e-prints, arXiv:1707.09322

Ahn, C. P., Alexandroff, R., Allende Prieto, C., et al. 2014, ApJS, 211, 17

Barentsen, G., Farnhill, H. J., Drew, J. E., et al. 2014, MNRAS, 444,3230

Becker, R. H., White, R. L., \& Helfand, D. J. 1995, ApJ, 450, 559

Capak, P., Aussel, H., Ajiki, M., et al. 2007, ApJS, 172, 99

Chambers, K. C., Magnier, E. A., Metcalfe, N., et al. 2016, ArXiv e-prints, arXiv: 1612.05560

Condon, J. J., Cotton, W. D., Greisen, E. W., et al. 1998, AJ, 115,1693

de Jong, J. T. A., Kleijn, G. A. V., Erben, T., et al. 2017, A\&A, 604, A134

Gaia Collaboration, Brown, A. G. A., Vallenari, A., et al. 2018, ArXiv e-prints, arXiv:1804.09365

-. 2016, A\&A, 595, A2

Helou, G., Madore, B. F., Bicay, M. D., Schmitz, M., \& Liang, J. 1990, in Astrophysics and Space Science Library, Vol. 160, Windows on Galaxies, ed. G. Fabbiano, J. S. Gallagher, \& A. Renzini, 109

Henden, A. A., Welch, D. L., Terrell, D., \& Levine, S. E. 2009, in American Astronomical Society Meeting Abstracts, Vol. 214, American Astronomical Society Meeting Abstracts \#214, 669

Ishihara, D., Onaka, T., Kataza, H., et al. 2010, A\&A, 514, A1

Jarrett, T. H., Chester, T., Cutri, R., et al. 2000, AJ, 119, 2498

Kunszt, P. Z., Szalay, A. S., Csabai, I., \& Thakar, A. R. 2000, in Astronomical Society of the Pacific Conference Series, Vol. 216 Astronomical Data Analysis Software and Systems IX, ed. N. Manset, C. Veillet, \& D. Crabtree, 141

Lawrence, A., Warren, S. J., Almaini, O., et al. 2007, MNRAS, 379,1599

Malkov, O., Dluzhnevskaya, O., Karpov, S., et al. 2012, Baltic Astronomy, 21, 319

Martin, D. C., Fanson, J., Schiminovich, D., et al. 2005, ApJ, 619, L1

Mazzarella, J. M., \& NED Team. 2017, in IAU Symposium, Vol. 325, Astroinformatics, ed. M. Brescia, S. G. Djorgovski, E. D. Feigelson, G. Longo, \& S. Cavuoti, 379-384

Meixner, M., Gordon, K. D., Indebetouw, R., et al. 2006, AJ, 132 2268

Motch, C.. \& Arches Consortium. 2015, in Astronomical Society of the Pacific Conference Series, Vol. 495, Astronomical Data Analysis Software an Systems XXIV (ADASS XXIV), ed. A. R. Taylor \& E. Rosolowsky, 437

Nieto-Santisteban, M. A., Thakar, A. R., Szalay, A. S., \& Gray, J. 2006, in Astronomical Society of the Pacific Conference Series, Vol. 351, Astronomical Data Analysis Software and Systems XV, ed. C. Gabriel, C. Arviset, D. Ponz, \& S. Enrique, 493
Ochsenbein, F., Bauer, P., \& Marcout, J. 2000, A\&AS, 143, 23

Ofek, E. O. 2014, MATLAB package for astronomy and astrophysics, Astrophysics Source Code Library, ascl:1407.005

Ofek, E. O., Laher, R., Surace, J., et al. 2012, PASP, 124, 854

Ogle, P. M., Mazzarella, J., Ebert, R., et al. 2015, in Astronomical Society of the Pacific Conference Series, Vol. 495, Astronomical Data Analysis Software an Systems XXIV (ADASS XXIV), ed. A. R. Taylor \& E. Rosolowsky, 25

Pineau, F.-X., Boch, T., \& Derriere, S. 2011, in Astronomical Society of the Pacific Conference Series, Vol. 442, Astronomical Data Analysis Software and Systems XX, ed. I. N. Evans, A. Accomazzi, D. J. Mink, \& A. H. Rots, 85

Ramírez, S. V., Arendt, R. G., Sellgren, K., et al. 2008, ApJS, 175,147

Riccio, G., Brescia, M., Cavuoti, S., et al. 2017, PASP, 129, 024005

Rosen, S. R., Webb, N. A., Watson, M. G., et al. 2016, A\&A, 590, A1

Shanks, T., Metcalfe, N., Chehade, B., et al. 2015, MNRAS, 451, 4238

Skrutskie, M. F., Cutri, R. M., Stiening, R., et al. 2006, AJ, 131, 1163

Szalay, A. S., Gray, J., Fekete, G., et al. 2007, eprint arXiv:cs/0701164, cs/0701164

Taylor, M. B. 2005, in Astronomical Society of the Pacific Conference Series, Vol. 347, Astronomical Data Analysis Software and Systems XIV, ed. P. Shopbell, M. Britton, \& R. Ebert, 29

Taylor, M. B. 2006, in Astronomical Society of the Pacific Conference Series, Vol. 351, Astronomical Data Analysis Software and Systems XV, ed. C. Gabriel, C. Arviset, D. Ponz, \& S. Enrique, 666

Voges, W., Aschenbach, B., Boller, T., et al. 2000, VizieR Online Data Catalog, 9029

Wenger, M., Ochsenbein, F., Egret, D., et al. 2000, A\&AS, 143, 9

Whitmore, B. C., Allam, S. S., Budavári, T., et al. 2016, AJ, 151, 134

Wright, E. L., Eisenhardt, P. R. M., Mainzer, A. K., et al. 2010, AJ, 140, 1868

Zacharias, N., Finch, C. T., Girard, T. M., et al. 2013, AJ, 145, 44 